\title{
A Study on Impact of Liquidity on Returns of Four Government Bonds in the Context of Indian Bond Market
}

\author{
Hima Vincent \\ Prajyoti Niketan College, Pudukkad, Kerala, India \\ E-Mail: himamanjaly@gmail.com
}

\begin{abstract}
A well-developed capital market consists of equity and bond market. A sound bond market with a significant role played by the Government bond market segment is considered to be important for an efficient capital market and raising for developmental ventures. Bonds are issued and sold to the public for funds. Bonds are interest bearing debt certificates. This study is conducted in order to analyze the impact of liquidity on return of government securities in the context of Indian bond market.

Keywords: Bond Market, Bond, Bond Yield, Liquidity Amihud Mendelson Model, Linear Regression.
\end{abstract}

\section{INTRODUCTION}

The Indian financial system is changing fast, marked by strong economic growth, more robust markets, and considerably greater efficiency. While the government and corporate bond markets have grown in size, they remain illiquid. The bond market, in addition, restricts participants and is largely arbitrage-driven.

To meet the needs of its firms and investors, the bond market must therefore evolve. The bond market in India has diversified to a large extent and that is a huge contributor to the stable growth of the economy. The debt market in India consists of mainly two categories the government securities or the G-Sec markets comprising central government and state government securities, and the corporate bond market.

In order to finance its fiscal deficit, the government floats fixed income instruments and borrows money by issuing GSec that are sovereign securities issued by the Reserve Bank of India (RBI) on behalf of the Government of India.

\section{STATEMENT OF THE PROBLEM}

The study is conducted to analyze the relationship between the two variables that is liquidity and its impact on the returns of selected government bonds. Liquidity is only variable that have to be studied here irrespective of many other factors affecting the returns of government bonds which could be the part of the model to better understand the returns. This study has been done in order to gain further insight and assessing the results.

\section{SIGNIFICANCE OF THE STUDY}

The significance of the study is that there are very less number of work done on this area. So as to verify the association among liquidity and bond return in Indian context in order to create awareness about different characteristics of bond market. An investor can reach to better decision by analyzing various factors affecting the return and liquidity of the bond. By knowing these factors investor may opt or not for the same.

\section{OBJECTIVES OF THE STUDY}

1. To analyze the relationship between bond yield and liquidity of selected government bonds.

2. To measure the average yield in the last four years and to assess Amihud's price impact factor of liquidity on the of selected government bonds.

\section{RESEARCH DESIGN}

This study is based on the market data relating to four government bonds collected from NSE for the period of 4 years (2013-16). A clear analysis has been made with the help of analytical tools, graphs and tables which provide useful and meaningful information.

Mean, standard deviation, coefficient of variation, Regression analyses were done to identify the relationship between return and liquidity in this study.

\section{SCOPE OF THE STUDY}

The scope is limited to the period from 2013 to 2016 of four government bonds collected from NSE. 


\section{DATA ANALYSIS AND INTERPRETATION}

TABLE I GOVERNMENT BOND 7.46\%

\begin{tabular}{|c|c|c|c|c|c|c|c|}
\hline Date & $\begin{array}{c}\text { Security } \\
\text { Type }\end{array}$ & $\begin{array}{c}\text { Security } \\
\text { Name }\end{array}$ & $\begin{array}{c}\text { Issue } \\
\text { Name }\end{array}$ & $\begin{array}{c}\text { Traded } \\
\text { Value }\end{array}$ & $\begin{array}{c}\text { Low Price/ } \\
\text { Rate }\end{array}$ & $\begin{array}{c}\text { High Price/ } \\
\text { Rate }\end{array}$ & LTP \\
\hline 29-Apr-13 & GS & CG2017 & $7.46 \%$ & 20 & 99.52 & 99.52 & 99.52 \\
\hline 22-May-13 & GS & CG2017 & $7.46 \%$ & 10 & 100.3797 & 100.3797 & 100.3797 \\
\hline 5-Sep-13 & GS & CG2017 & $7.46 \%$ & 5 & 95.5799 & 95.5799 & 95.5799 \\
\hline 10-Sep-13 & GS & CG2017 & $7.46 \%$ & 5 & 95.527 & 95.527 & 95.527 \\
\hline 20-Nov-13 & GS & CG2017 & $7.46 \%$ & 10 & 96.6855 & 96.6855 & 96.6855 \\
\hline 3-Mar-14 & GS & CG2017 & $7.46 \%$ & 5 & 96.051 & 96.051 & 96.051 \\
\hline 5-Sep-14 & GS & CG2017 & $7.46 \%$ & 10 & 97.4538 & 97.4538 & 97.4538 \\
\hline 7-Nov-14 & GS & CG2017 & $7.46 \%$ & 25 & 98.2123 & 98.2123 & 98.2123 \\
\hline 26-Nov-14 & GS & CG2017 & $7.46 \%$ & 25 & 98.3 & 98.3 & 98.3 \\
\hline 10-Dec-14 & GS & CG2017 & $7.46 \%$ & 25 & 98.783 & 98.783 & 98.783 \\
\hline
\end{tabular}

TABLE II 7.49\%

\begin{tabular}{|c|c|c|c|c|c|c|c|}
\hline Date & $\begin{array}{c}\text { Security } \\
\text { Type }\end{array}$ & $\begin{array}{c}\text { Security } \\
\text { Name }\end{array}$ & $\begin{array}{c}\text { Issue } \\
\text { Name }\end{array}$ & $\begin{array}{c}\text { Traded } \\
\text { Value }\end{array}$ & $\begin{array}{c}\text { Low Price/ } \\
\text { Rate }\end{array}$ & $\begin{array}{c}\text { High Price/ } \\
\text { Rate }\end{array}$ & LTP \\
\hline 25-Feb-13 & GS & CG2017 & $7.49 \%$ & 100 & 98.734 & 98.734 & 98.734 \\
\hline 6-Mar-13 & GS & CG2017 & $7.49 \%$ & 10 & 98.68 & 98.68 & 98.68 \\
\hline 12-Mar-13 & GS & CG2017 & $7.49 \%$ & 95 & 98.5109 & 98.5109 & 98.5109 \\
\hline 13-Mar-13 & GS & CG2017 & $7.49 \%$ & 5 & 98.6 & 98.6 & 98.6 \\
\hline 21-Mar-13 & GS & CG2017 & $7.49 \%$ & 15 & 98.5 & 98.5211 & 98.5211 \\
\hline 22-Mar-13 & GS & CG2017 & $7.49 \%$ & 5 & 98.4 & 98.4 & 98.4 \\
\hline 9-Apr-13 & GS & CG2017 & $7.49 \%$ & 75 & 98.845 & 99.08 & 99.08 \\
\hline 17-Apr-13 & GS & CG2017 & $7.49 \%$ & 25 & 99.2551 & 99.2551 & 99.2551 \\
\hline 21-Jun-13 & GS & CG2017 & $7.49 \%$ & 40 & 99.46 & 99.46 & 99.46 \\
\hline 28-Jun-13 & GS & CG2017 & $7.49 \%$ & 5 & 99.2 & 99.2 & 99.2 \\
\hline
\end{tabular}

TABLE III 7.99\%

\begin{tabular}{|c|c|c|c|c|c|c|c|}
\hline Date & $\begin{array}{c}\text { Security } \\
\text { Type }\end{array}$ & $\begin{array}{c}\text { Security } \\
\text { Name }\end{array}$ & $\begin{array}{c}\text { Issue } \\
\text { Name }\end{array}$ & $\begin{array}{c}\text { Traded } \\
\text { Value }\end{array}$ & $\begin{array}{c}\text { Low Price/ } \\
\text { Rate }\end{array}$ & $\begin{array}{c}\text { High Price/ } \\
\text { Rate }\end{array}$ & LTP \\
\hline 3-Jan-13 & GS & CG2017 & $7.99 \%$ & 10 & 99.99 & 99.99 & 99.99 \\
\hline 9-Jan-13 & GS & CG2017 & $7.99 \%$ & 5 & 100.18 & 100.18 & 100.18 \\
\hline 17-Jan-13 & GS & CG2017 & $7.99 \%$ & 35 & 100.33 & 100.33 & 100.33 \\
\hline 22-Feb-13 & GS & CG2017 & $7.99 \%$ & 100 & 100.495 & 100.495 & 100.495 \\
\hline 7-Mar-13 & GS & CG2017 & $7.99 \%$ & 420 & 100.34 & 100.39 & 100.36 \\
\hline 15-Mar-13 & GS & CG2017 & $7.99 \%$ & 15 & 100.46 & 100.46 & 100.46 \\
\hline 19-Mar-13 & GS & CG2017 & $7.99 \%$ & 15 & 100.31 & 100.31 & 100.31 \\
\hline 20-Mar-13 & GS & CG2017 & $7.99 \%$ & 100 & 100.18 & 100.18 & 100.18 \\
\hline 21-Mar-13 & GS & CG2017 & $7.99 \%$ & 50 & 100.18 & 100.18 & 100.18 \\
\hline
\end{tabular}


TABLE IV $8.07 \%$

\begin{tabular}{|c|c|c|c|c|c|c|c|}
\hline Date & Security Type & Security Name & Issue Name & Traded Value & Low Price/Rate & High Price/Rate & LTP \\
\hline 2-Jan-13 & GS & CG2017 & $8.07 \%$ & 10 & 100.46 & 100.46 & 100.46 \\
\hline 18-Jan-13 & GS & CG2017 & $8.07 \%$ & 60 & 100.84 & 100.84 & 100.84 \\
\hline 21-Jan-13 & GS & CG2017 & $8.07 \%$ & 25 & 100.5682 & 100.5682 & 100.5682 \\
\hline 22-Jan-13 & GS & CG2017 & $8.07 \%$ & 35 & 100.6096 & 100.6096 & 100.6096 \\
\hline 30-Jan-13 & GS & CG2017 & $8.07 \%$ & 100 & 100.65 & 100.65 & 100.65 \\
\hline 6-Feb-13 & GS & CG2017 & $8.07 \%$ & 200 & 100.3574 & 100.3574 & 100.3574 \\
\hline 18-Feb-13 & GS & CG2017 & $8.07 \%$ & 100 & 100.5 & 100.5 & 100.5 \\
\hline 20-Feb-13 & GS & CG2017 & $8.07 \%$ & 125 & 100.65 & 100.65 & 100.65 \\
\hline 22-Feb-13 & GS & CG2017 & $8.07 \%$ & 100 & 100.735 & 100.778 & 100.778 \\
\hline 14-Mar-13 & GS & CG2017 & $8.07 \%$ & 50 & 100.569 & 100.569 & 100.569 \\
\hline 15-Mar-13 & GS & CG2017 & $8.07 \%$ & 20 & 100.715 & 100.715 & 100.715 \\
\hline
\end{tabular}

A. Return Has Been Calculated In Here by the Following Formula

Return $=\mathrm{P}_{1}-\mathrm{P}_{0} / \mathrm{P}_{0}$ Liquidity is calculated by ABS return/value traded $* 10^{7}$

TABLE V 7.46\% BOND

\begin{tabular}{|c|c|c|}
\hline Date & Return & Illiquidity \\
\hline 29-Apr-13 & 0 & 0 \\
\hline 22-May-13 & 0.008638465 & 0.086384646 \\
\hline 5-Sep-13 & 0.047816441 & 0.956328819 \\
\hline 10-Sep-13 & 0.000553464 & 0.011069273 \\
\hline 20-Nov-13 & 0.012127461 & 0.121274613 \\
\hline 3-Mar-14 & 0 & 0 \\
\hline 5-Sep-14 & 0.014604741 & 0.146047412 \\
\hline 7-Nov-14 & 0.007783175 & 0.031132701 \\
\hline 26-Nov-14 & 0.000892964 & 0.003571854 \\
\hline 10-Dec-14 & 0.00491353 & 0.01965412 \\
\hline
\end{tabular}

TABLE VII 7.46\% BOND

\begin{tabular}{|c|c|c|}
\hline Date & Absolute Return & Amihud Illiquidity \\
\hline 25-Feb-13 & 0 & $0.00 \mathrm{E}+00$ \\
\hline 6-Mar-13 & 0.000546924 & 0.005469241 \\
\hline 12-Mar-13 & 0.00171362 & 0.00180381 \\
\hline 13-Mar-13 & 0.000904468 & 0.018089369 \\
\hline 21-Mar-13 & 0.000800203 & 0.005334686 \\
\hline 22-Mar-13 & 0.001229178 & 0.024583566 \\
\hline 9-Apr-13 & 0.006910569 & 0.009214092 \\
\hline 17-Apr-13 & 0.001767259 & 0.007069035 \\
\hline 21-Jun-13 & 0.002064378 & 0.005160944 \\
\hline 28-Jun-13 & 0.002614116 & 0.052282325 \\
\hline
\end{tabular}

\section{B. Regression Analysis}

Regression analysis can be analyzed to know about the relationship between liquidity and return and to know the significance of return and illiquidity.

\begin{tabular}{|c|c|c|c|c|c|c|}
\hline \multicolumn{7}{|c|}{ Coefficient } \\
\hline & \multirow{2}{*}{ Model } & \multicolumn{2}{|c|}{ Unstandardized Coefficients } & \multirow{2}{*}{$\begin{array}{c}\text { Standardized Coefficients } \\
\text { Beta } \\
\end{array}$} & \multirow{2}{*}{$\mathbf{T}$} & \multirow{2}{*}{ Sig. } \\
\hline & & B & Std. Error & & & \\
\hline \multirow[b]{2}{*}{1} & (Constant) & .002 & .001 & & 2.646 & .014 \\
\hline & $\begin{array}{l}\text { Amihud } \\
\text { Illiquidity }\end{array}$ & .051 & .004 & .925 & 12.162 & .000 \\
\hline
\end{tabular}

TABLE VIII 7.49\% BOND

\begin{tabular}{|c|c|c|c|c|c|c|}
\hline \multicolumn{7}{|c|}{ Coefficient } \\
\hline \multirow{2}{*}{\multicolumn{2}{|c|}{ Model }} & \multicolumn{2}{|c|}{ Unstandardized Coefficients } & \multirow{2}{*}{$\begin{array}{c}\text { Standardized Coefficients } \\
\text { Beta } \\
\end{array}$} & \multirow{2}{*}{$\mathbf{t}$} & \multirow{2}{*}{ Sig. } \\
\hline & & B & Std. Error & & & \\
\hline \multirow{2}{*}{1} & (Constant) & .002 & .001 & & 4.033 & .000 \\
\hline & AmihudIlliquidity & .024 & .011 & .234 & 2.260 & .026 \\
\hline \multicolumn{7}{|c|}{ a. Dependent Variable: AbsoluteReturn } \\
\hline
\end{tabular}


TABLE IX 7.99\% BOND

\begin{tabular}{|c|c|c|c|c|c|c|}
\hline \multicolumn{7}{|c|}{ Coefficient } \\
\hline \multirow{2}{*}{\multicolumn{2}{|c|}{ Model }} & \multicolumn{2}{|c|}{ Unstandardized Coefficients } & \multirow{2}{*}{$\begin{array}{c}\text { Standardized Coefficients } \\
\text { Beta }\end{array}$} & \multirow{2}{*}{$\mathbf{t}$} & \multirow{2}{*}{ Sig. } \\
\hline & & B & Std. Error & & & \\
\hline \multirow{2}{*}{1} & (Constant) & .002 & .000 & & 4.313 & .000 \\
\hline & AmihudIlliquidity & .070 & .012 & .551 & 5.948 & .000 \\
\hline \multicolumn{7}{|c|}{ a. Dependent Variable: AbsoluteReturn } \\
\hline
\end{tabular}

TABLE X 8.07\% BOND

\begin{tabular}{|c|c|c|c|c|c|c|}
\hline \multicolumn{7}{|c|}{ Coefficient } \\
\hline \multirow{2}{*}{\multicolumn{2}{|c|}{ Model }} & \multicolumn{2}{|c|}{ Unstandardized Coefficients } & \multirow{2}{*}{$\begin{array}{c}\text { Standardized Coefficients } \\
\text { Beta }\end{array}$} & \multirow{2}{*}{$\mathbf{t}$} & \multirow{2}{*}{ Sig. } \\
\hline & & B & Std. Error & & & \\
\hline \multirow{2}{*}{1} & (Constant) & .002 & .000 & & 4.001 & .000 \\
\hline & AmihudIlliquidity & .109 & .019 & .512 & 5.690 & .000 \\
\hline \multicolumn{7}{|c|}{ Dependent Variable: Absolute Return } \\
\hline
\end{tabular}

It means illiquidity decreases, the return of bond increases. The higher beta coefficient of $7.46 \%$ bond compared to other three government bonds shows that the illiquidity has more impact on $7.46 \%$ bond than others.

\section{FINDINGS OF THE STUDY}

1. The Indian corporate bond market is in a developing stage.

2. The relationship between return and illiquidity is significant as per Amihud illiquidity measure so here the relationship between return and liquidity is also significant for all the four government bonds.

3. $7.46 \%$ bonds are more volatile and inconsistent as compared to other bonds.

4. Coefficient of variation is more for $7.99 \%$ bond (0.6016) that of $7.49 \%$ bond have $(0.5430)$ so $7.49 \%$ Bonds are more stable.

5. Illiquidity and return shows a positive relationship that is lower the return higher the illiquidity. This means there is a negative relationship between return and liquidity.

6. An investor aiming for a stable return should opt for $7.49 \%$ bonds over others.

7. The bond market still has to improve and thus it needs more growth like that of the stock market.

8. Range of return of bonds is more for $7.46 \%$ which shows more variation. Thus $7.49 \%$ is more stable and it is having only less range compared to other government bonds.

\section{CONCLUSION}

From this study we can reach to a conclusion that both the bonds are having significant relationship with return and liquidity and thus there exist a positive relationship between return and liquidity. For an investor to choose among these bonds he may opt for $7.49 \%$ bond over $7.46 \%$, $7.99 \%$, $8.07 \%$ bonds because they are volatile and is inconsistent compared to the other. $7.49 \%$ security here shows more consistency and stability than others. So for regular and high return investor can choose that security rather than others.

\section{REFERENCES}

[1] Akram, N. (2014). The Effect of Liquidity on Stock Returns: An Evidence From Pakistan. IOSR Journal of Business and Management.

[2] Alonso, F. (2006). Estimating liquidity premia in the Spanish government securities market. The European Journal of Finance.

[3] Dharman , A. (2017). Financil Management. ISRO, 5(8), 12.

[4] Dufour, A. (2011). Permanent trading impacts and bond yields. The European Journal of Finance.

[5] Gregoriou, A. (2011). The liquidity effects of revisions to the CAC40 stock index. Applied Financial Economics.

[6] Mahapatra, D. S. (2016). investor's attitude towards post office deposit schemes.

[7] Mahdi Salehi, G. T. (2011). A Study of the Relationship Between Liquidity and Stock Returns of Companies Listed in Tehran Stock Exchange. World Applied Sciences .

[8] Pynnonen, S. (2010). Expectations and Liquidity in Yen Bond Markets. Journal of the Asia Pacific Economy.

[9] Seth, R. (2010). A Study on Yield Spreads and Liquidity Measures in the Indian Bond Market. Calcutta: Indian Institute Of Management .

[10] Westerholm, J. (2002). The Relationship Between Liquidity, Trading Activity and Return - Studies of the Finnish and Swedish Stock Markets. Econ 